ONLINE MUTATION REPORT

\title{
Novel mutations in COX15 in a long surviving Leigh syndrome patient with cytochrome $c$ oxidase deficiency
}

\author{
M Bugiani, V Tiranti, L Farina, G Uziel, M Zeviani
}

J Med Genet 2005;42:e28 (http://www.jmedgenet.com/cgi/content/full/42/5/e28). doi: 10.1136/jmg.2004.029926

\begin{abstract}
Background: Isolated cytochrome $c$ oxidase (COX) deficiency is usually associated with mutations in several factors involved in the biogenesis of COX.

Methods: We describe a patient with atypical, long surviving Leigh syndrome carrying two novel mutations in the COX15 gene, which encodes an enzyme involved in the biosynthesis of heme A.

Results: Only two COX15 mutated patients, one with severe neonatal cardiomyopathy, the other with rapidly fatal Leigh syndrome, have been described to date. In contrast, our patient had a slowly progressive course with no heart involvement. COX deficiency was mild in muscle and a normal amount of fully assembled COX was present in cultured fibroblasts.

Conclusions: The clinical and biochemical phenotypes in COX15 defects are more heterogeneous than in other conditions associated with COX deficiency, such as mutations in SURF1.
\end{abstract}

\footnotetext{
C
} ytochrome $c$ oxidase (COX), the terminal component of the mitochondrial respiratory chain, catalyses the exergonic reduction of molecular oxygen to water. COX is a heteromeric complex consisting of three catalytic subunits, encoded by mitochondrial DNA (mtDNA), and ten "accessory" subunits, encoded by nuclear genes. The latter are presumably involved in the structural stabilisation and assembly of the complex, and in the modulation of its catalytic activity. Different clinical phenotypes associated with COX deficiency have been described, generally displaying an early onset of symptoms and a fatal outcome. Maternally inherited mutations in mtDNA encoded COX subunits are rare. Most of the syndromes are inherited as autosomal recessive traits. In several of these cases, the responsible gene remains unknown. No mutation in any of the nucleus encoded COX subunits has yet been identified. By contrast, all the genetically defined cases so far reported are due to mutations in genes encoding factors involved in the biogenesis and assembly of the complex, including SURF1, COX10, SCO1, SCO2 and, more recently, COX15. Mutations in the SURFI gene, consistently associated with typical Leigh syndrome, are by far the most prevalent cause of infantile COX deficiency, while mutations in the other nuclear genes involved in COX biogenesis are much less common. ${ }^{12}$

We report on a child with Leigh syndrome and isolated COX deficiency, in whom the biochemical defect is due to two different novel mutations in the COX15 assembly gene. The clinical presentation is different from the rapidly progressive course of previous cases, as the patient is still surviving at 16 years of age.

\section{CASE REPORT}

The proband is the third born of unrelated Italian parents; two older sisters are healthy. From the first days of life he displayed poor sucking, feeding difficulties, and failure to thrive. A severe psychomotor delay was noted at 4 months of age, with diffuse hypotonia and muscle wasting and weakness. Subsequently he developed brisk deep tendon reflexes, cerebellar tremor, and eye movement incoordination. A first MRI at 18 months of age showed symmetric signal changes in the posterior part of the putamina and bilateral cerebellar white matter abnormalities. Lactate and pyruvate were markedly elevated in plasma (lactate $3.6 \mathrm{mM}$, normal values (nv) 0.5-1.8; pyruvate $0.19 \mathrm{mM}$, nv 0.06-0.13) and in CSF (lactate $3.8 \mathrm{mM}$, nv $0.8-2.0$; pyruvate $0.17 \mathrm{mM}$, nv 0.045-0.125). Clinical and MRI features were consistent with the diagnosis of Leigh syndrome. Symptoms slowly worsened thereafter with virtual arrest of body growth, progressive loss of postural control, and onset of dystonic postures in the upper limbs, while the cognitive functions remained relatively better preserved. A follow up MRI study at 4 years of age disclosed a mild progression of the cerebellar white matter and putaminal lesions, and the appearance of bilateral signal changes in the head of the caudate nuclei (fig 1). His clinical features remained grossly unchanged thereafter, although plasma lactate and pyruvate progressively decreased to normal levels. Apart from the central nervous system and skeletal muscle, the patient showed no abnormality in other tissues or organs, including the heart, gastrointestinal tract, liver, kidneys, and haemopoietic system. The boy is now 16 years old.

\section{METHODS}

Morphological and biochemical analyses

Morphological analysis of skeletal muscle and biochemical assays of the individual respiratory chain complexes on muscle homogenate and cultured skin fibroblasts were carried out as described. ${ }^{3}$ Enzymatic activity of each complex was normalised to that of citrate synthase.

\section{Genetic analysis}

Molecular analysis was performed on genomic DNA extracted from cultured fibroblasts. Overlapping fragments of the COX15 coding region were PCR amplified and products were assessed on agarose gel, purified, and sequenced according to Antonicka et $a l^{4}$ with slight modifications.

\section{Western blot analysis of protein fraction resolved by 2D-BNE}

For two dimensional Blue Native electrophoresis (2D-BNE), crude mitochondrial pellets, obtained as described, ${ }^{5}$ were resuspended in $100 \mu \mathrm{l}$ of $1.5 \mathrm{M}$ 6-aminohexanoic acid, $50 \mathrm{mM}$ Bis-Tris, pH 7.0. Then $20 \mu \mathrm{l}$ of $10 \% \beta$-lauryl maltoside

Abbreviations: 2D-BNE, two dimensional Blue Native electrophoresis; COX, cytochrome $c$ oxidase; mtDNA, mitochondrial DNA 

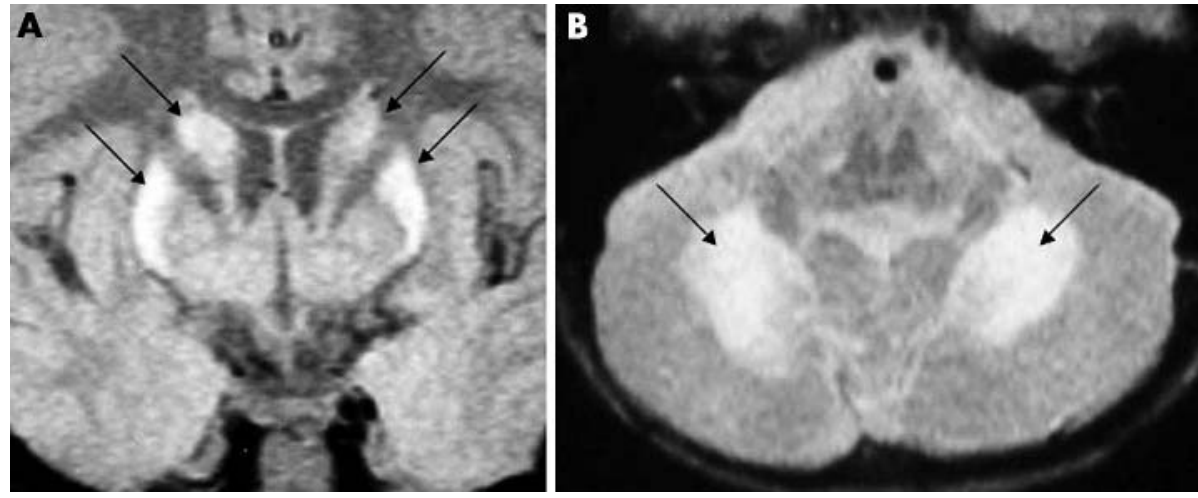

Figure 1 Brain MRI. (A) Coronal proton density weighted image shows bilateral lesions in the putamina and in the head of caudate nuclei (arrows). (B) Axial T2 weighted image shows extensive cerebellar white matter involvement (arrows).

were added and the samples were incubated for 15 min on ice. Clearing of the samples was performed by centrifugation at $12000 \times g$ for $20 \mathrm{~min}$ at $4^{\circ} \mathrm{C}$. The supernatant was removed and $10 \mu \mathrm{l}$ of $5 \%$ Serva Blue G in 1 M 6-aminohexanoic acid was added prior to loading. 2D-BNE and electroblotting procedures were performed as described. ${ }^{6}$ The blots were immunostained using mouse monoclonal antibodies raised against bovine COX subunit I (Molecular Probes, Eugene, OR), as previously described. ${ }^{5}$

\section{RESULTS AND DISCUSSION}

A muscle biopsy, performed at the age of 18 months, was morphologically normal. Biochemical assays (table 1) showed an isolated COX deficiency both in skeletal muscle and cultured fibroblasts $(42 \%$ and $22 \%$ residual activity compared to the mean of controls, respectively). Accordingly, the cytochemical reaction to COX was moderately reduced in cultured fibroblasts as compared to normal control cells, but much stronger than that observed in fibroblasts from SURFI mutant Leigh syndrome patients (fig 2).

Mutation analysis revealed a compound heterozygous state for two novel mutations in the COX15 gene. These mutations were absent in 40 alleles from ethnically matched controls, and are not listed in any SNP database. The first mutation, a $503 \mathrm{C} \rightarrow \mathrm{G}$ transversion, introduces a premature stop codon in exon $4(\mathrm{H} 152 \mathrm{X}){ }^{7}$ The second mutation, a 1081T $\rightarrow \mathrm{C}$ missense transition in exon 8 , results in a serine to proline substitution at amino acid position 344, in the C-terminal end of the fifth predicted transmembrane domain of the protein. The S344 residue is conserved from vertebrates to Neurospora crassa, and is conservatively replaced by a threonine in a few species such as Drosophila melanogaster, an arthropod animal, and Arabidopsis thaliana, a vascular plant.

In humans, alternative splicing of the COX15 gene generates two transcript variants (COX15.1 and COX15.2; GenBank accession numbers NM 078470 and NM 004376) diverging in the $3^{\prime}$-UTR region and in the predicted Cterminal amino acid sequence. The functional significance of the two splice variants is still unknown. ${ }^{8}$ However, the mutations in our case affect the portion of the COX15 amino acid sequence that is common to both variants. COXI5 is a membrane bound mitochondrial protein involved in the last part of the biosynthetic pathway of heme A, an essential step in the biogenesis of COX. Studies on yeast mutants indicate that COX15 is part of a three component mono-oxygenase, catalysing the hydroxylation of the methyl group at position 8 of heme $\mathrm{O}$, the precursor molecule of heme A. Heme $\mathrm{O}$ is the product of the farnesylation of pro-heme (heme B), a reaction catalysed by COX10, another enzyme involved in the early steps of COX formation. The alcohol group added to heme $\mathrm{O}$ is then oxidised into the corresponding aldehyde by an unknown enzyme, to eventually produce heme A. Accordingly, yeast COX15 mutants have undetectable levels of heme A and detectable levels of heme O. ${ }^{9-11}$

To study the assembly of COX in our COX15 mutant patient, we performed Western blot analysis on mitochondrial proteins extracted from cultured fibroblasts and separated by 2D-BNE. In contrast with the accumulation of COX assembly intermediates, which is typical of SURF1 defective mitochondria, ${ }^{5}$ the COXI5 mutant mitochondria contained a virtually normal amount of apparently fully assembled COX complex (fig 2).

Besides our patient, COX15 mutations have previously been reported in only two other cases (table 2 ). Albeit rare, defects in this gene appear to be associated with considerable clinical and biochemical heterogeneity. The first child presented soon after birth with muscle hypotonia, epilepsy, and lactic acidosis, but the clinical course was dominated by the development of a rapidly fatal hypertrophic cardiomyopathy. ${ }^{12}$ The COX residual activity was markedly reduced in heart tissue $(6 \%)$ and was low $(25-30 \%)$ in liver, kidney, and cultured fibroblasts but normal in skeletal muscle. The child was a compound heterozygote with a missense mutation $(700 \mathrm{C} \rightarrow \mathrm{T}, \mathrm{R} 217 \mathrm{~W})$ in exon 5 on one allele and a splice site mutation (C447-3G) in intron 3 on the other allele, resulting in the deletion of exon 4 . Interestingly, the BNE pattern obtained from heart mitochondria was similar to that of our proband, that is, fully assembled COX, with no assembly intermediates, ${ }^{4}$ although the amount of crossreacting material was much lower, in agreement with the

Table 1 Biochemical activities*

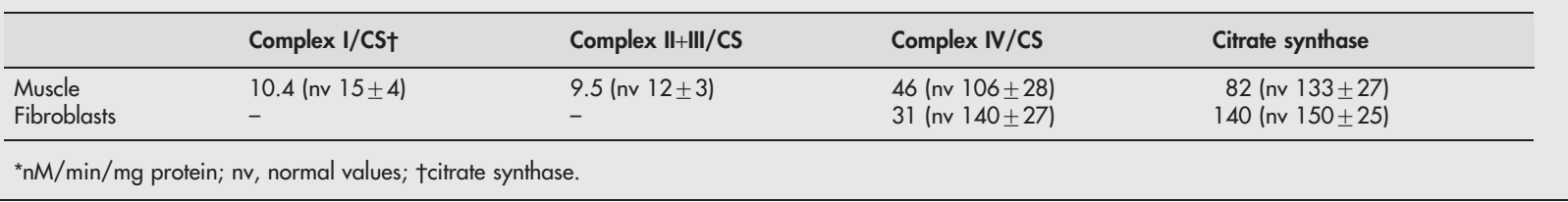


Table 2 Clinical features of COX15 mutated patients

\begin{tabular}{|c|c|c|c|}
\hline & $\begin{array}{l}\text { Kennaway et } a l^{12} \\
\text { Antonicka et } a l^{4}\end{array}$ & Oquendo et $a l^{13}$ & Present case \\
\hline $\begin{array}{l}\text { Gender } \\
\text { Family history } \\
\text { Age at onset/at death } \\
\text { Neurological signs }\end{array}$ & $\begin{array}{l}\text { Female } \\
- \\
\text { Birth/24 days } \\
\text { Hypotonia } \\
\text { Epileptic seizures }\end{array}$ & $\begin{array}{l}\text { Male } \\
\overline{7} \\
7 \text { months } / 3 \text { years } 11 \text { months } \\
\text { Axial hypotonia } \\
\text { Limb spasticity } \\
\text { Bulbar signs } \\
\text { Nystagmus, retinopathy microcephaly }\end{array}$ & $\begin{array}{l}\text { Male } \\
- \\
4 \text { months/still alive (16 years) } \\
\text { Axial hypotonia } \\
\text { Limb spasticity } \\
\text { Cerebellar tremor } \\
\text { Eye movement incoordination } \\
\text { Dystonia }\end{array}$ \\
\hline Hypertrophic cardiomyopathy & + & - & - \\
\hline Other clinical features & Midfacial hypoplasia & Gastrointestinal dysfunction & Somatic growth below 3rd centile \\
\hline Plasma lactate (mM) & Increased & 5.2 & 3.6 \\
\hline CSF lactate (mM) & NS & 6.2 & 3.8 \\
\hline Brain MRI & NS & $\begin{array}{l}\text { Basal nuclei } \\
\text { Brainstem tegmentum } \\
\text { Cerebral peduncles }\end{array}$ & $\begin{array}{l}\text { Basal ganglia (putamina, caudate) } \\
\text { Cerebellar white matter }\end{array}$ \\
\hline Mutations & $700 C \rightarrow T, C 447-3 G$ & $700 \mathrm{C} \rightarrow \mathrm{T}, 700 \mathrm{C} \rightarrow \mathrm{T}$ & $503 C \rightarrow G, 1081 T \rightarrow C$ \\
\hline
\end{tabular}

NS, not specified in the report.

extreme reduction of COX activity in cardiac mitochondria. Similar to yeast COX15 mutants, the cytochrome spectrum showed low levels of heme A and accumulation of heme O.

More recently, the same $700 \mathrm{C} \rightarrow \mathrm{T}$ missense mutation (R217W) has been reported in a homozygous child with rapidly progressive Leigh syndrome and chronic gastrointestinal dysfunction, who died at 4 years of age. The R217 is a highly conserved residue located in the region between the third and the fourth transmembrane domain of the protein. Residual COX activity in cultured fibroblasts was undetectable by both spectrophotometric and cytochemical assays. ${ }^{13}$

By contrast, the clinical course in our patient was slow, with partial preservation of the cognitive functions and survival beyond adolescence. The residual COX activity in muscle and fibroblasts was only moderately reduced; this could in turn account for the slower clinical progression.

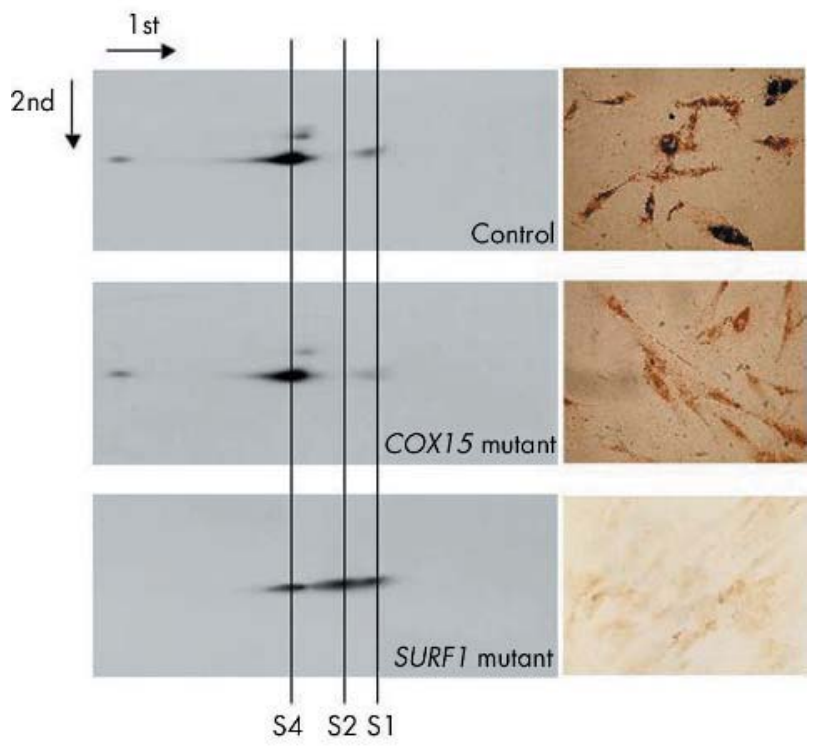

Figure 2 Analysis of COX. Left panel: Immunoblot staining of 2D-BNE using an anti-COX I specific monoclonal antibody. The analysis was performed on cultured skin fibroblasts from a control, from our COX 15 patient, and from a SURF ${ }^{-1-}$ patient. S1 and S2 are assembly intermediates of the COX holoenzyme, corresponding to S4. The arrows indicate the direction of the electrophoretic run in the first and second dimensions, respectively. Right panel: COX cytochemical staining of the cultured fibroblast lines used for the 2D-BNE immunoblot analysis.
However, wide tissue variability of the biochemical defect was observed also in the previous two COX15 mutant cases; therefore, a mild reduction of COX activity in muscle and fibroblasts does not exclude a more severe biochemical defect in other tissues, including the brain. An interesting possibility is that the S344P change has a hypomorphic, rather than null, effect, determining a partial reduction, rather than abolition, of the COX15 catalytic activity on heme A biosynthesis. This hypothesis could also explain the presence of a normal amount of fully assembled COX found in our patient, in contrast with the lower than normal amount found in the first COX15 mutant case, and also in other defects affecting the heme A biosynthesis, notably mutations in the COXIO gene. ${ }^{14}$

\section{Authors' affiliations}

M Bugiani, G Uziel, Department of Child Neurology, Istituto Nazionale Neurologico "C. Besta", Milan, Italy

V Tiranti, M Zeviani, Department of Molecular Neurogenetics, Istituto Nazionale Neurologico "C. Besta", Milan, Italy

L Farina, Department of Neuroradiology, Istituto Nazionale Neurologico "C. Besta", Milan, Italy

This work was supported by Fondazione Telethon-ltaly (grant no. GGP030039), Fondazione Pierfranco e Luisa Mariani, Ricerca Finalizzata Ministero della Salute FR-2002/158, Fondazione Cariplo, and a EUMITOCOMBAT network grant from the European Union Framework Program 6.

Competing interests: none declared

Correspondence to: Massimo Zeviani, Department of Molecular Neurogenetics, Istituto Nazionale Neurologico "C. Besta", via Libero Temolo, 420126 Milano, Italy; zeviani@istituto-besta.it

Revised version received 10 December 2004

Accepted for publication 17 December 2004

\section{REFERENCES}

1 Shoubridge E. Cytochrome c oxidase deficiency. Am J Med Genet 2001; 106:46-52.

2 Sacconi S, Salviati L, Sue CM, Shanske S, Davidson MM, Bonilla E, Naini AB, de Vivo DC, Dimauro S. Mutation screening in patients with isolated cytochrome c oxidase deficiency. Pediatr Res 2003;53:224-30.

3 Tiranti V, Munaro M, Sandona D, Lamantea E, Rimoldi M, DiDonato S, Bisson R, Zeviani M. Nuclear DNA origin of cytochrome $c$ oxidase deficiency in Leigh's syndrome: genetic evidence based on patient's-derived rho degrees transformants. Hum Mol Genet 1995;4:2017-23.

4 Antonicka H, Mattman A, Carlson CG, Glerum DM, Hoffbuhr KC, Leary SC, Kennaway NG, Shoubridge EA. Mutations in COX15 produce a defect in the mitochondrial heme biosynthetic pathway, causing early-onset fatal hypertrophic cardiomyopathy. Am J Hum Genet 2003;72:101-14 
5 Tiranti V, Galimberti G, Niitmans L, Bovolenta S, Perini MP, Zeviani M Characterization of SURF-1 expression and Surf- $1 \mathrm{p}$ function in normal and disease conditions. Hum Mol Genet 1999;8:2533-40.

6 Nijtmans LG, Henderson NS, Holt IJ. Blue Native electrophoresis to study mitochondrial and other protein complexes. Methods 2002;26:327-34.

7 Jacobson A, Peltz SW. Interrelationships of the pathways of mRNA decay and translation in eukaryotic cells. Annu Rev Biochem 1999;65:693-739.

8 Petruzzella V, Tiranti V, Fernandez $P$, lanna $P$, Carrozzo R, Zeviani M Identification and characterization of human CDNAs specific to BCS1, PET1 12, SCO1, COX15, and COX11, five genes involved in the formation and function of the mitochondrial respiratory chain. Genomics 1998;54:494-504.

9 Barros MH, Carlson CG, Glerum DM, Tzagoloff A. Involvement of mitochondrial ferredoxin and Cox15p in hydroxylation of heme O. FEBS Lett $2001 ; 492: 133-8$
10 Barros MH, Nobrega FG, Tzagoloff A. Mitochondrial ferredoxin is required for heme A synthesis in Saccharomyces cervisiae. J Biol Chem 2002;277:9997-10002

11 Barros MH, Tzagoloff A. Regulation of the heme A biosynthetic pathway in Saccharomyces cervisiae. FEBS Lett 2002;516:119-23.

12 Kennaway NG, Carrero-Valenzuela RD, Ewart G, Balan VK, Lightowlers R, Zhang YZ, Powell BR, Capaldi RA, Buist NR. Isoforms of mammalian cytochrome c oxidase: correlation with human cytochrome $c$ oxidase deficiency. Pediatr Res 1990;28:529-35.

13 Oquendo CE, Antonicka H, Shoubridge EA, Reardon W, Brown GK Functional and genetic studies demonstrate that mutation in the COX15 gene can cause Leigh syndrome. J Med Genet 2004;41:540-4.

14 Coenen MJH, van den Heuvel LP, Ugalde C, ten Brinke M, Nijtmans LGJ Trijbels FJM, Beblo S, Maier EM, Muntau AC, Smeitink JAM. Cytochrome oxidase biogenesis in a patient with a mutation in COX10 gene. Ann Neurol 2004;56:560-4. 